\title{
Effects of gender, age and menopausal status on serum apolipoprotein concentrations
}

\author{
Panagiotis Anagnostis ${ }^{1,2}$, John C. Stevenson ${ }^{3}$, David Crook ${ }^{4}$, Desmond G. Johnston ${ }^{1}$,lan F. Godsland ${ }^{1}$
}

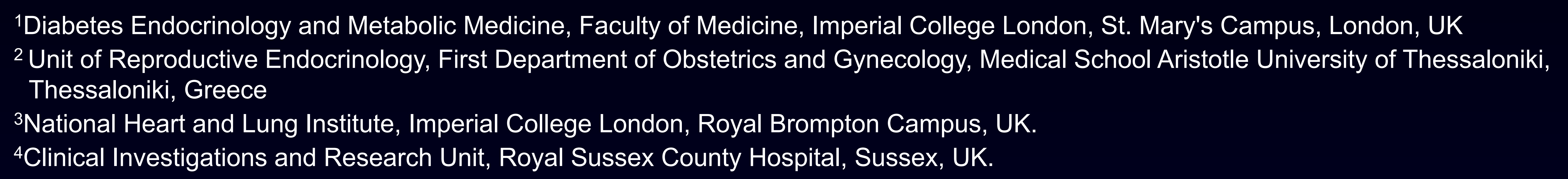

Introduction: Inconsistent data exist as to whether menopause is associated with increased cardiovascular disease (CVD) risk. However, few studies have evaluated differences in apolipoprotein metabolism according to menopausal status and gender. The aim of this study was to investigate the effects of gender and menopause on serum apolipoprotein B (apoB), A-I (apoA-I) and A-II (apoA-II) concentrations.

Methods: A cross-sectional analysis was undertaken of age and gender-related differences in apparently healthy Caucasian premenopausal and postmenopausal women not taking oral contraceptives or hormone replacement, and Caucasian men. Measurements included serum apoA-I, apoAII, apoB, total cholesterol, low-density and high-density lipoprotein cholesterol (LDL-C and HDL-C respectively), triglycerides, cholesterol in HDL subfractions 2 and 3 and the apoB/apoA-I, LDL-C/apoB, HDL-C/apoA-I and $\mathrm{HDL}-\mathrm{C} / \mathrm{apoA}-\mathrm{II}$ ratios. Analyses were undertaken with and without standardization for confounding characteristics and in 5 year age ranges.

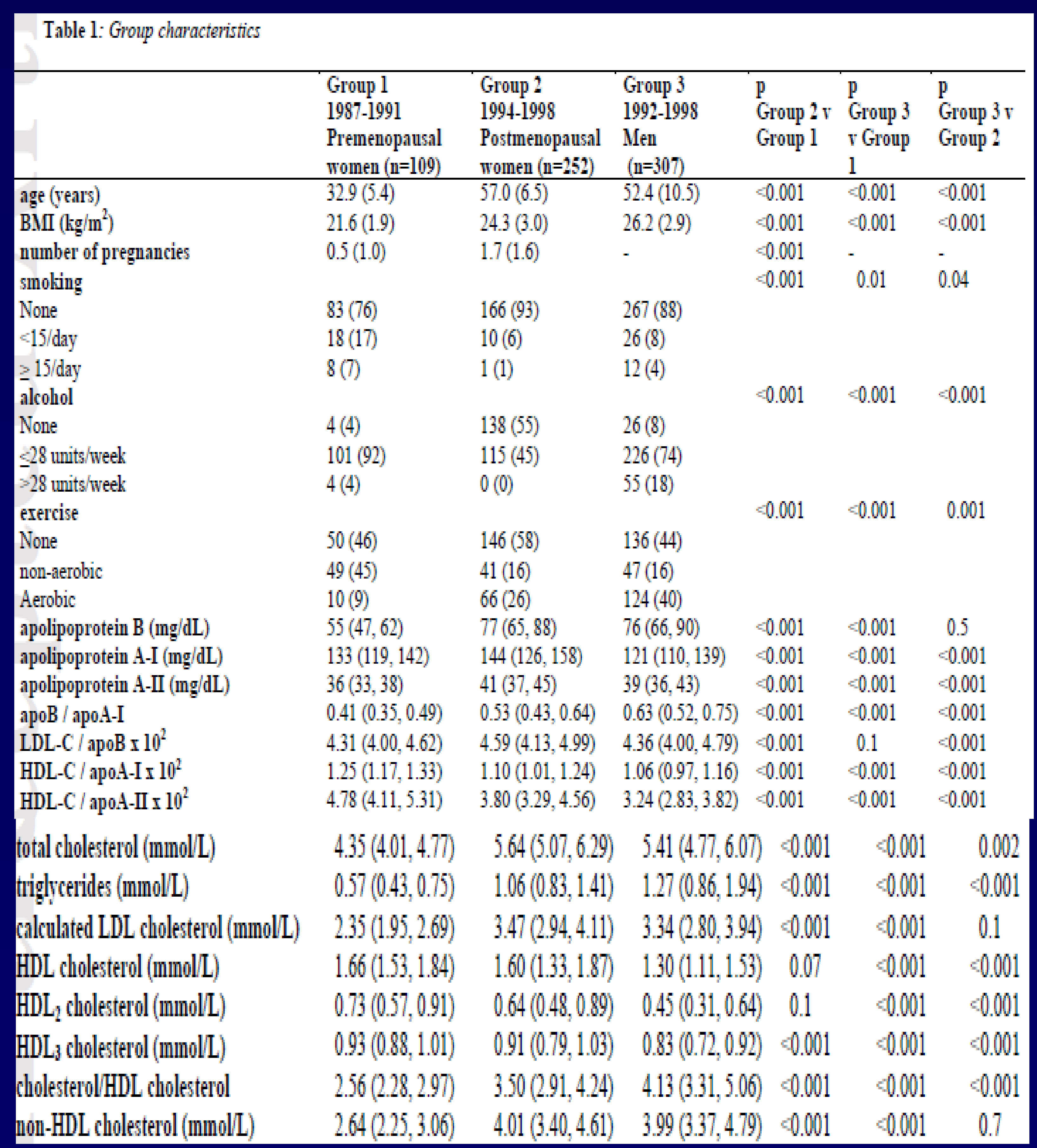

References:

Anagnostis P, et al. Maturitas 2015;81: 62-8, Godsland IF, et al. J Clin Endocrinol Metab. 1992;74:64-70, Godsland IF, et al. Clin Endocrinol (Oxf). 2004;60:541-549 Bittner V. Am Coll Cardiol. 2009;54:2374-2375.
Results: 109 pre-menopausal women (aged 32.9 \pm 5.4 years), 253 post-menopausal women (aged $57 \pm 6.5$ years) and 307 men (aged $52.4 \pm 10.5$ years) were included in the analysis.

$>$ Overall, apoB concentrations were highest in men but rose with age and menopause in women to converge with concentrations in men in the age range 50-55 years.

$>$ The LDL-C/apoB ratio was generally higher in women than men, especially postmenopausally.

$>$ Both apoA-I and apoA-II concentrations were highest in postmenopausal women and lowest in men.

$>$ Men generally had the lowest ratios of HDL-C to apoA-I and to apoA-II, but the highest ratios were apparent in premenopausal women. I

$>\mathrm{n}$ multivariable analyses, incorporating age, BMI, smoking, alcohol, exercise and number of pregnancies, the above differences were sustained.

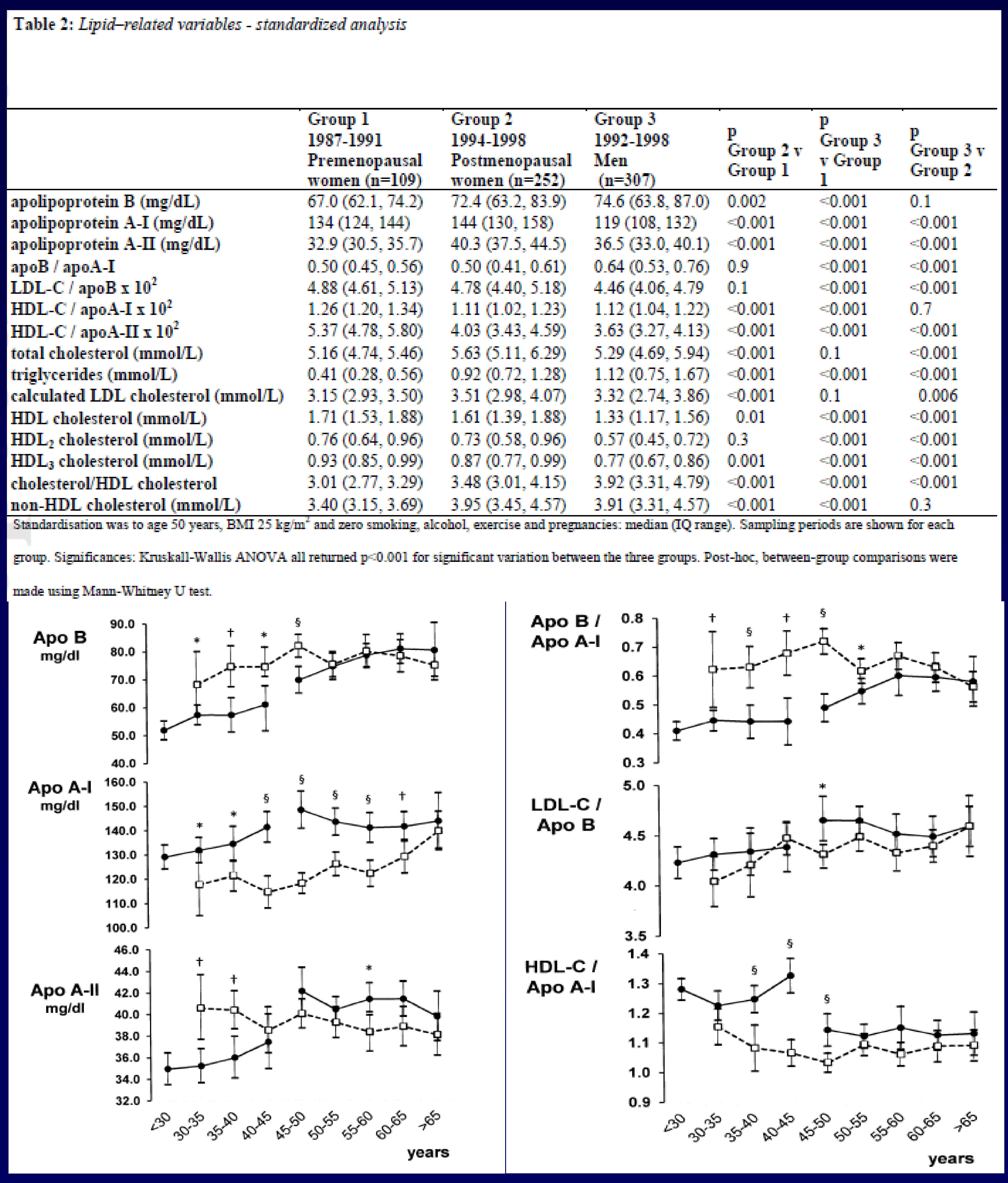

Conclusions: Adverse effects of ageing in women, male gender and menopause on apoB concentrations and of menopause and, in particular, male gender on the cholesterol content of HDL particles were consistent with adverse effects on CVD risk, with male gender having the greatest effect. 